\title{
The High Output Maximum Efficiency Resonator (HOMER) Developed for Long Life, Space-Based Altimetry
}

\author{
D. Barry Coyle \\ NASA-GSFC \\ Code 690.1 \\ Greenbelt, MD 20771 \\ 301-614-5859 \\ barry@cornfed.gsfc.nasa.gov
}

\author{
Paul R. Stysley \\ The American Univ. \\ Dept. of Physics \\ Washington, DC 20016 \\ 301-614-5863 \\ paul@cornfed.gsfc.nasa.gov
}

Abstract-We report on the status of the High Output Maximum Efficiency Resonator (HOMER), a prototype space-flight $\mathrm{Nd}$ :YAG, diode pumped, unstable resonator. HOMER, the $3^{\text {rd }}$ generation of this design based on the High Efficiency Laser Transmitter, is a stand-alone oscillator that has achieved $\sim 2$ billion $10 \mathrm{~ns}$ q-switched pulses at 17-15 mJ. In this configuration HOMER achieves an excellent optical efficiency of $\sim 16 \%$. It is currently the lead candidate for the Biomass Monitoring Mission (BioMM); a global vegetation instrument currently under design and preproposal at NASA-GSFC.

\section{TABLE OF CONTENTS}

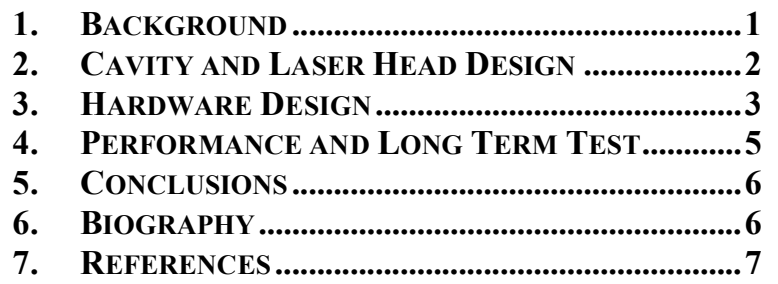

\section{BACKGROUND}

NASA-GSFC is actively pursuing laser-based altimetry methods for earth and planetary remote sensing instruments. The laser technology must prove to be reliable, efficient, robust, and long-lived before deployment on a spacecraft is deemed feasible. The High Efficiency Laser Transmitter (HELT), shown in Figure 1, is a conductively-cooled laser featuring a high efficiency pump head employed in a concave-convex unstable resonator with a graded reflectivity mirror (GRM) output coupler. [1] The GRM is used to achieve near-TEM $\mathrm{oo}_{\mathrm{o}}$ output and produce large beam sizes in concert with the cavity's radially expanding beam and relatively small cavity length. [2] HELT was developed in order to achieve efficient, damage-free operation at $15 \mathrm{~mJ}$ in a $\mathrm{TEM}_{00}$ mode, while using the minimum number of components. This system showed much promise as a flight laser candidate with immediate results in high efficiency and good beam quality. Other advantages of oscillator-only designs are revealed in cost, size, and mass. ${ }^{1,2}$

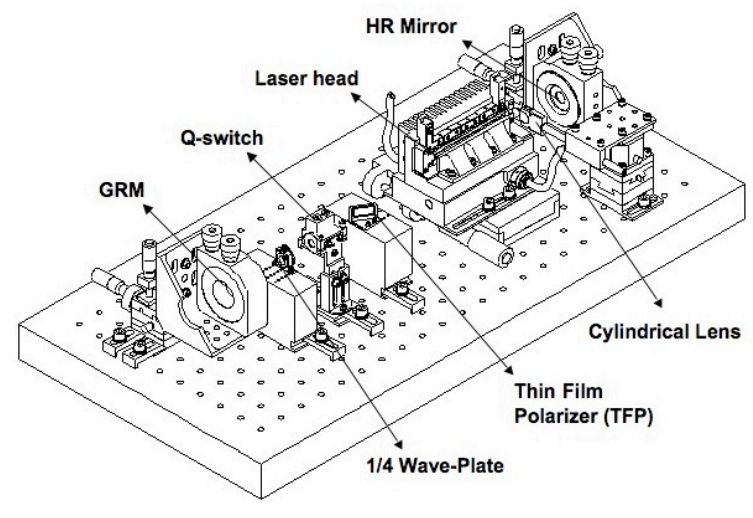

Figure 1 - The HELT breadboard was built to study the feasibility, mode quality, and efficiency of diode pumped $\mathrm{Nd}$ :YAG slab geometries in unstable resonators.

The HELT laser operated continuously, without damage, for more than $4.8 \times 10^{9}$ shots in a test in 2002. [3] A $2^{\text {nd }}$ generation of HELT was reconfigured in an attempt to fit into the existing enclosure for the Vegetation Canopy Lidar (VCL) mission after the mission selected laser failed to meet specifications. [4] Based on HELT's success and lessons learned through the VCL project, we have constructed a $3^{\text {rd }}$ generation "hardened breadboard", or prototype, with certain experiments in mind such as vacuum operation, vibration, thermal vacuum, power cycling, and more life tests. We call this unit the High Output Maximum Efficiency Resonator (HOMER), shown in Figure 2. This version serves as a base design for the oscillator stage of the Laser Risk Reduction Program (LRRP) and as a lead candidate for the pre-proposed Biomass Monitoring Mission (BioMM), which requires the laser to fire approximately $2 \mathrm{x}$ $10^{9}$ laser pulses per year over 2 years. The operational specifications require a repetition rate of $242 \mathrm{~Hz}, 17-15 \mathrm{~mJ}$ pulse energy, and a pulsewidth of $\sim 10 \mathrm{~ns}$.

1 U.S. Government work not protected by U.S. copyright
${ }^{2}$ IEEEAC paper \#1001, Version 6, Updated Oct, 282005 


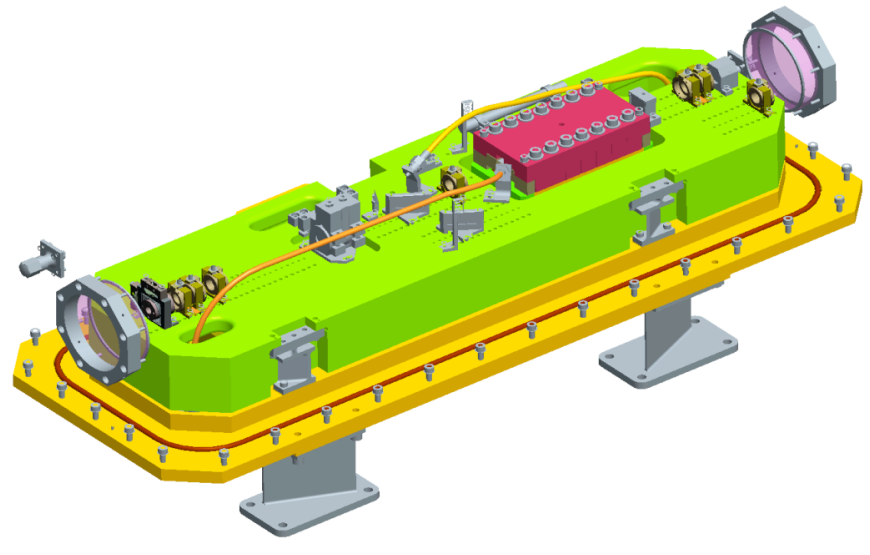

Figure 2 - The HOMER prototype is shown with an "invisible" lid, hence the floating output windows and pressure valves. This laser incorporated all we had learned with HELT and VCL into a hardened package capable of extensive environmental testing.

\section{Cavity and Laser Head Design}

The HOMER transmitter uses a positive branch unstable resonator (PBUR) cavity with a geometrical length of 41 $\mathrm{cm}$, with an effective $33 \%$ reflectivity GRM with a radius $\mathrm{R}_{\mathrm{GRM}}=-237 \mathrm{~cm}$ and a high reflector $(\mathrm{HR})$ end mirror of $\mathrm{R}_{\mathrm{HR}}$ $=+250 \mathrm{~cm}$. [5] The most important feature of an unstable resonator is its magnification, M. Cavity magnification directly affects the dissipative losses (feedback), higher order mode discrimination, alignment sensitivity, and the GRM reflective spot size relative to the beam waist in the active media. [1] Our GRM design resulted in a $1 / \mathrm{e}^{2}$ reflective spot size of $1.12 \mathrm{~mm}$ and a peak reflectivity of $~$ $65 \%$. This GRM design, when incorporated in this cavity, provided a total magnification (geometrical + diffraction) of $\mathrm{M}_{\mathrm{t}}=1.4$.

The packaged HOMER laser had some similarities to the $100 \mathrm{~mJ}$ high brightness design of Armandillo et.al., which was a side-pumped slab laser in a PBUR configuration as well. [6] The obvious differences between our design and that of Armandillo et al. are in output energy and laser head pumping geometry. Additionally, there are more subtle differences such as our use of an even-bounce slab with a $26.5^{\circ}$ tip angle, a stepped Copper:Tungsten slab heat sink for thermal lens reduction and good matching of Nd:YAG's coefficient of thermal expansion. A shorter resonator was used for mass and size reduction, and a large overhead of available pump LDA power to allow for long life and bar drops. Other diode side-pumped slab designs that have gone to space have not employed an unstable resonator but rather used porro or retro prisms as end mirrors for their expected high alignment stability and large multimode beam production. [7][8]

It was determined early on that the best way to efficiently produce $10-20 \mathrm{~mJ} \mathrm{TEM}_{00}$ pulses in an oscillator-only system, is with the use of a side pumped zig-zag slab configured in a large mode cavity. The slab geometry provides a longer gain length/pump length ratio and more effectively uses the pumped regions along the pump face than side pumped rods. The flat surfaces of slabs are also much easier to conductively cool without fluids. The zigzag path cancels optical distortion in the zig-zag plane (yaxis) to first order, and side-pumping in this plane takes advantage of the multi-stripe diode array geometry, allowing efficient pump energy coupling into the intra-slab mode volume. [9][10] HOMER's Nd:YAG slab is a 22bounce slab with near-Brewster end faces, a center length of $\sim 90 \mathrm{~mm}$ along its optical z-axis, a thickness (pump depth) of $2.65 \mathrm{~mm}$, (y-axis), and a $5 \mathrm{~mm}$ width (x-axis). It is coated along the long faces with AR and HR dielectrics to produce a 2-pass pump geometry at $809 \mathrm{~nm}$. The slab is suspended by a thermal conductive adhesive adhered to the HR surface over seven 4-bar, Quasi-CW (QCW) laser diode arrays, each rated at $60 \mathrm{~W} / \mathrm{bar}$. These LDAs were selected to have $\sim 50 \%$ electrical-to-optical efficiency upon procurement, and were typically operated at $20-30 \%$ below the peak output power in order to extend their operational lifetime reduce the expected decay rates. After extensive modeling and laboratory studies, we have optimized, for this cavity, the pumping geometry and electrical drive parameters to produce the maximum efficiency and largest cavity mode possible.

The laser head has evolved into a carefully crafted assembly where the components are pinned and fastened to minimize thermo-mechanical distortions with good symmetrical heat removal. The unassembled laser head is detailed in Figures 3 and 4.

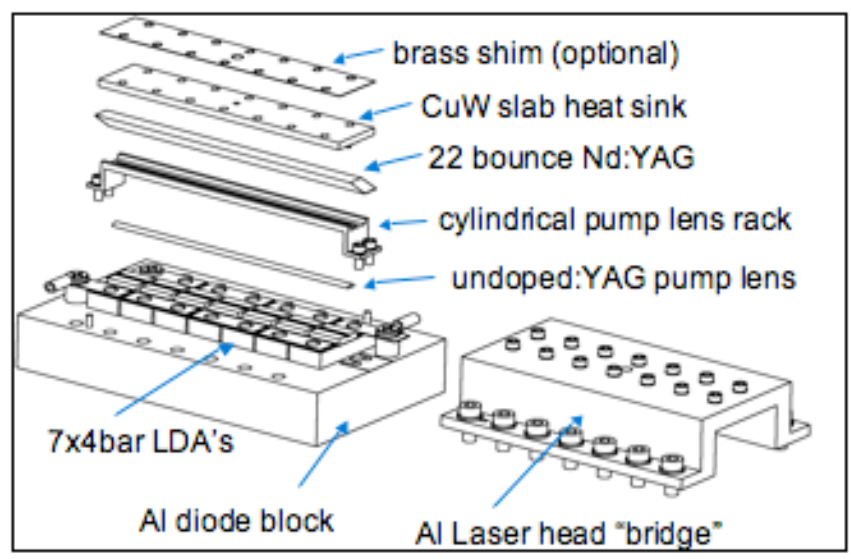

Figure 3 - Component breakdown of our laser head assembly. The "brass shim" and pump lens shim (not shown) are eventually removed for final manufacture and assembly.

The resonator efficiency depends strongly on the location, shape, and value of the inversion density produced. Thus, it is also advantageous to further confine the pump light in the transverse dimension such that it matches our cavity mode as closely as possible. However, if the pump beam is 
confined into too small of a volume, strong thermal effects can disrupt the cavity parameters, cause misalignment, self focusing, and eventual optical damage. To optimize the pumped volume to the oscillator cavity beam, the $809 \mathrm{~nm}$ LDA light is simply "collimated" in the fast axis by a single plano-convex cylindrical lens made of undoped YAG. A significant effort has been made to produce a pumped region that optimally matches the cavity mode and creates the smoothest intensity distribution possible across the slab interior.

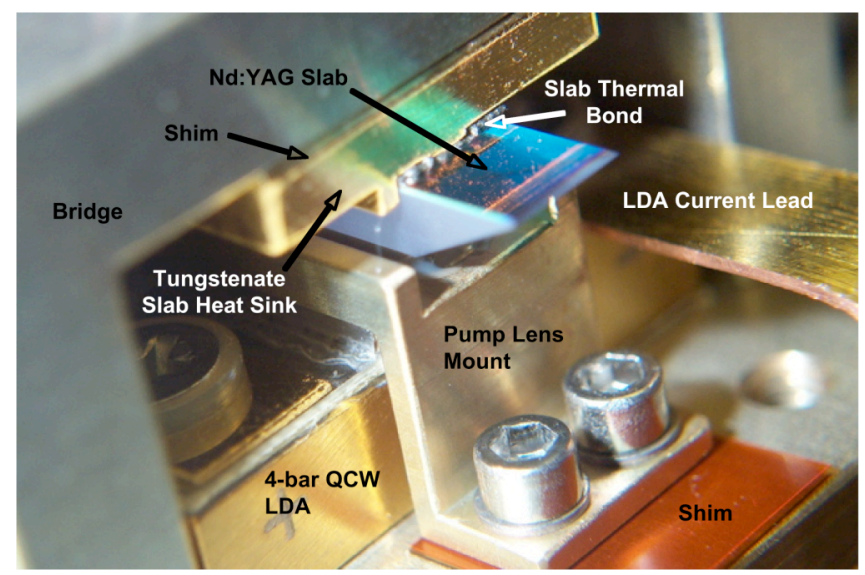

Figure 4 - Detailed view of the assembled head with optimization shims installed. The near-Brewster tipped $\mathrm{Nd}$ :YAG slab extends from the head and is not heat sunk to reduce end effects.

Our $3^{\text {rd }}$ generation modular laser head assembly performs very well with high efficiency, low thermal lensing, high stability, and excellent mechanical repeatability. The precise modular nature of this head has been critical in its development since we were able to perform frequent microscopic inspections of all the head components between developmental verification and operational tests. This head design also incorporates specific points to accept shim material with high precision in order to characterize the relative positions with accuracies of $\pm 5 \mathrm{um}$ and provided a method in which to verify, quantify, and optimize our ray tracing and mathematical pumping models. Once the LDA/pump-lens/slab positions are all experimentally optimized, the pump lens mount and slab heat sink are readily re-manufactured to these shimmed dimensions and installed in the final laser.

Furthermore, this pumping scheme produced higher efficiencies than one using a close-coupled, unlensed sidepumped slab. Our unlensed pumping tests typically produced less thermal lensing but could not meet our output specifications with proper LDA derating. The YAG lens greatly increased the optical efficiency by creating an intense stripe of pump energy down the slab length, maximizing overlap between the inversion density and cavity mode.

Care must be taken, however, to not increase the risk of opto-mechanical failure by adding optics to the pumping scheme or optical damage on the slab. Damage on the slab's pump surface has been created in the past with and without a pump lens. The damage was due to overstressing the AR coating with a combination of high $809 \mathrm{~nm}$ pump intensities, corresponding localized high inversion densities, and high fluence of the $1064 \mathrm{~nm}$ cavity mode. Furthermore, the risk of added optics in the laser system is also negated by the creation of a particulate shield or "sneeze guard" which protects the slab. We have demonstrated several times, especially over long term runs, that diode array sputter and spewed semiconductor particulates would have deposited on the Nd:YAG's pump face and possibly caused contamination damage on the slab's AR coating if not for the pump lens' presence.

Our QCW LDAs were procured from CEO (Cutting Edge Electronics, a division of Northrop Grumman) but diodes with similar short-term performance can be obtained from several vendors. The SDL (Spectra Diode Labs) LDAs we have on the shelf are approaching 10 years old, thus we needed to upgrade to current technologies and higher efficiencies. The $\mathrm{CEO}$ diodes were specified to operate at a higher temperature of about $35^{\circ} \mathrm{C}$ where the SDL diodes ran at $25^{\circ} \mathrm{C}$. This applies less thermal load requirements to the enclosure base. The temperature set point is simply a tradeoff between required cooling capacity to maintain a temperature regime and the lifetime effects of running diodes slightly warm. In our characterization tests of the LDAs, we discovered several interesting issues.

We discovered that the linewidth of these diode stacks were much narrower than the SDL arrays, as seen in Figure 5. On one hand, this reveals that more absorption occurs at the slab pump face and therefore leads to stronger thermal lensing. This also means that the diodes must run within a tighter temperature range. We monitor the laser to be sure that the diodes run within $1{ }^{\circ} \mathrm{C}$ of the quoted optimum operating temperature. On the other hand a higher effective absorption is achieved and thus, higher laser efficiency. This is advantageous only if adequate thermal lens correction is possible. The narrower linewidth also allows for greater derating for the LDAs while still producing the required system output powers. The pros and cons of the linewidth issues and their effects on flight laser instrument design are still under study.

\section{HARDWARE Design}

The hardware produced for HOMER is based somewhat on the VCL mechanics and have been incorporated where possible to reduce cost and design time. Typical re-used components included cavity optics, mounts, flexures, feedthrough electrical and fiber connections, and output windows. Everything else was redesigned and manufactured to incorporate as many improvements as determined over the post VCL studies. Besides the head design discussed earlier, other important improvements involved the enclosure and the optical bench. These 


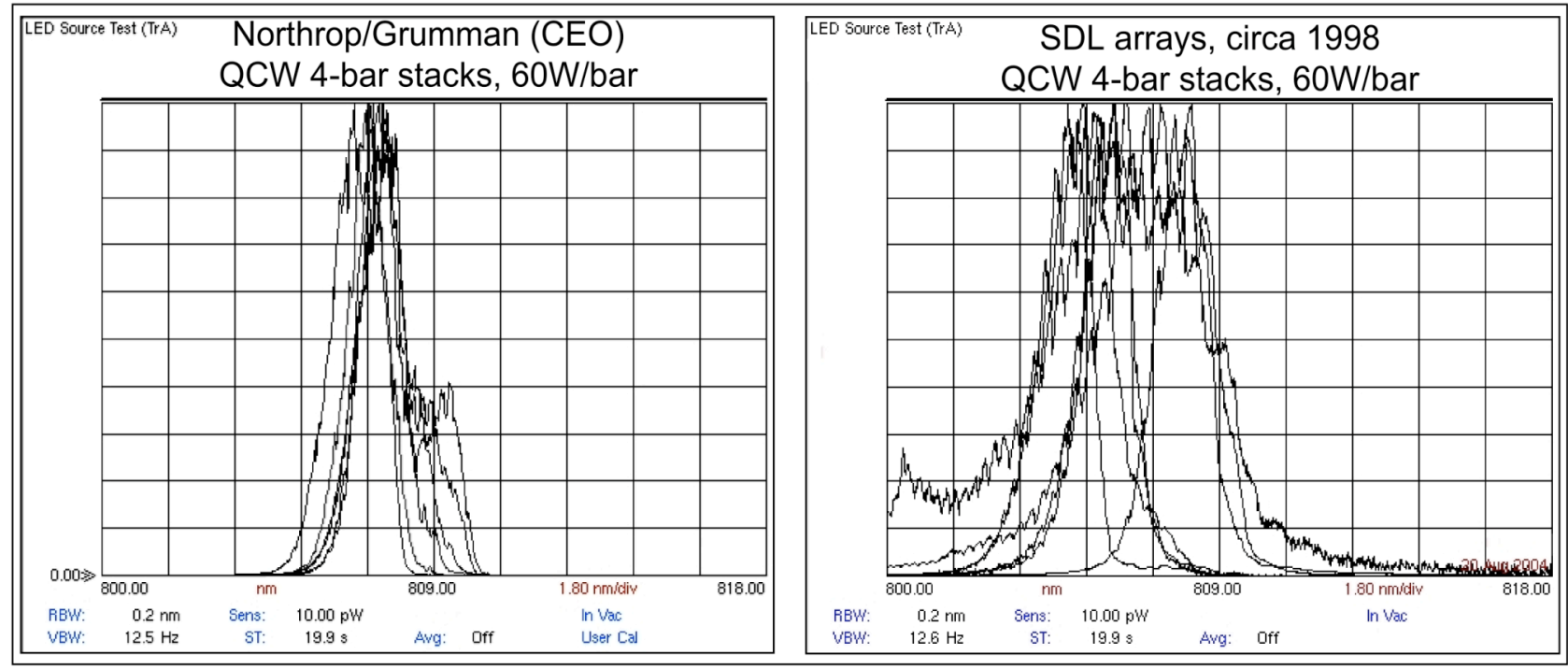

Figure 5 - Measured linewidths of 4-bar laser diode array G-packages from SDL and CEO. Both are shown centered about $808 \mathrm{~nm}$, but have a temperature roughly $10^{\circ} \mathrm{C}$ apart.

upgrades can be envisioned as a sealed butter dish where the enclosure is the "lid" and the optical bench is the "plate". This design allows for easy access to all the optics, adjustments, and fasteners upon laser assembly, while providing a strong enclosure for hermetic sealing.

The use of two opposing windows in the enclosure allows for the output beam to emerge at one end of the box, as well as the ability to image the intracavity beam through the cavity's HR mirror at the other end. By monitoring the intra-cavity beam fluence during operation, we can determine and track, with great accuracy and safety, any cavity mode effects due to environmental events such as opto-mechanical movements, thermal lens variations, and LDA output variations.

The optical bench is simply an inverted $4 \mathrm{~cm}$ deep "bucket" with a rectangular opening for the laser head to emerge and position the slab in the optical path. The bench is isolated thermally and kinematically "floating" on 3 titanium flexures and therefore not directly contacted to the Nd:YAG laser head. The laser head bridge assembly is mated to the diode array "pedestal", which is also thermally fastened and pinned to the enclosure's base plate. There are several interfaces in this design which raises the effective steady state temperature difference between the Nd:YAG pump face and the underside of the enclosure baseplate where the heat is finally removed. However, we have found that a warm slab ( $\sim 41^{\circ} \mathrm{C}$ ) has little or no effect on the laser performance or lifetime, and actually reduces the uniaxial thermal lens which resides within. However, these interfaces are all precision to provide the modularity required for frequent inspections and component changes if necessary without the need for cavity realignment. When operating at $242 \mathrm{~Hz}$ and $17 \mathrm{~mJ}$ pulse energy, BioMM requirements, we calculate a thermal load of $\sim 40 \mathrm{~W}$ into the base of the pedestal. Little or no heat is allowed to migrate to the optical bench since the system heat sink, or cold plate, is bolted directly below the LDA pedestal location. This water-cooled plate simulates a spacecraft's conductive cooling radiator or heat sink, and actually must be heated to about $31^{\circ} \mathrm{C}$ to achieve optimal laser performance in the laboratory. Were our water "chiller" to fail and the laser allowed to continue operation, the laser temperature would initially drop closer to room temperature.

The Q-switch driver used was a commercial OEM board from Analog Modules, and housed in a shielded subenclosure under the optical bench and below the Q-Switch cell. We used an off-the-shelf $\mathrm{KD}^{*} \mathrm{P}$ device from Cleveland Crystals, with a $1 / 4$ wave voltage of $\sim 3.2 \mathrm{kV}$, in combination with a $1 / 4$ wave plate to produce our $10 \mathrm{~ns}$ pulses. Two pair of $1^{\circ}$ risley prisms are employed at each cavity end for optical alignment and the HR mirror is housed in a static mount. As a risley pair provides pointing (pitch and yaw) of each end mirror, the GRM output coupler also requires $\mathrm{X}$ and $\mathrm{Y}$ translation such that the Gaussian reflective spot remains co-centered with the laser cavity beam. This iterative process of pointing and translation is required with the addition of each optic in the laser during assembly and alignment. An acceptable flight quality translational mount could not be found commercially during assembly, thus we had to produce a design in-house. No less than 4 versions, including one commercial design with some alterations, are under development and are all being studied in a parallel effort to determine which exhibits the best survivability for flight, as well as adequate usability for alignment. The GRM mount design in this HOMER build was selected simply because it was the first one available for use at the time and has performed well in our initial tests to date. 


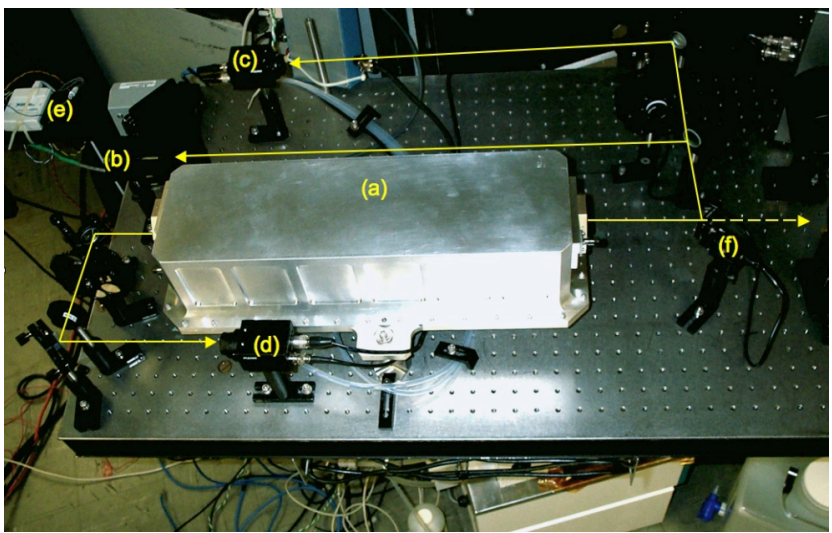

Figure 6 - Lifetime experiment setup: (a) sealed HOMER laser. Output beam exiting to the right and a $0.1 \%$ HR beam to the left. (b) Optical power meter, (c) far field CCD camera via a $1 \%$ pickoff optic and fold mirror, (d) fluence monitor CCD camera, (e) A/D box for laser temperatures and voltages, and (f) flip mirror for use of HOMER output for MLA flyby experiment.

\section{Performance and Long Term Test}

Once assembled it was found that this laser was an excellent candidate for a laser ranging experiment with the Mercury Laser Altimeter (MLA) aboard the Messenger spacecraft as it performed an Earth "flyby" in late May 2005 on its way to Mercury. [11] In order for the MLA data system to successfully capture a laser pulse from HOMER, it had to operate at $240 \mathrm{~Hz}$ and produce more than $15 \mathrm{~mJ}$. We took this small funding opportunity to perform a 3 month operational test in situ. Thus, HOMER was installed at the $1.2 \mathrm{~m}$ ranging telescope facility, based at Goddard's Geophysical and Astronomical Observatory (GGAO). Operating in an automated eyesafe setup with proper interlocks, HOMER was turned on and left to run continuously while accumulating housekeeping and performance data under careful automation. Our data system recorded power output, laser temperatures, near field and far field beam images. Also a $90^{\circ} \mathrm{HR}$ flip mirror was deployed in the immediate output beam, as seen in our experimental setup in Figure 6, which allowed the MLA scientists to gain access to the laser output at their convenience and schedule. Thus, during the numerous MLA ranging tests, no HOMER performance data was gathered.

HOMER was initially driven with a diode pulsewidth of 85 us and a current of $50 \mathrm{~A}$. This provided an $\mathrm{X}$ and $\mathrm{Y}$ beam divergence of $1.1 \mathrm{uRad}$ and $1.0 \mathrm{uRad}$, respectively. The near field $1 / \mathrm{e}^{2}$ beam diameter was somewhat elliptical at the GRM output, which is typical for theses systems, and measured $1.75 \mathrm{~mm}$ and $2.26 \mathrm{~mm}$ (X and $\mathrm{Y}$ ). Using these numbers we calculate the $\mathrm{X}$ and $\mathrm{Y} \mathrm{M}^{2}$ values of 1.43 and 1.67, respectively. Our experience has shown repeatedly over the years that PBUR cavities with a GRM and $\mathrm{Nd}$ :YAG slab configuration usually give filamentary near field beam images which make calculating beam sizes and fluences "best effort" measurements. We also recorded the beam size just inside the cavity at the HR mirror in order to track intracavity fluences. This beam size was typically $1.7 \mathrm{~mm}$ in the $\mathrm{X}$-axis and $2.2 \mathrm{~mm}$ in the $\mathrm{Y}$-axis. Sample beam images from HOMER can be seen in Figure 7.

In July 2005, HOMER successfully pulsed $\sim 2$ billion shots with an energy output of 17-15 mJ and a Q-switched output pulse of $10 \mathrm{~ns}$. Though we are confidant that HOMER could have achieved more shots, the lifetest was ended when the MLA flyby experiment was concluded due to limited funding and prior commitments. We are especially pleased with this result in that the output energy degradation over time of $0.94 \mathrm{~mJ} / 10^{9}$ shots is a vast improvement over a $4.8 \times 10^{9}$ shot lifetest performed on the HELT breadboard version in late 2002 through early 2003. [1] This output, combined with the reported $\mathrm{HR}$ beam sizes, gives acceptable fluences of below $2.5 \mathrm{~J} / \mathrm{cm}^{2}$ and optical efficiencies as high as $16.5 \%$. During the test we did witness some anomalies, which were somewhat expected. The final results of this test are summarized in Figure 8

The LDA pulsewidth and LDA drive current were individually increased on two occasions to maintain pulse energies $>16 \mathrm{~mJ}$ and to see if either pump adjustment

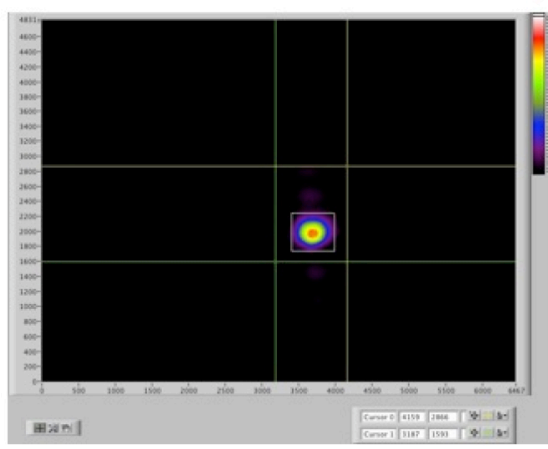

(a)

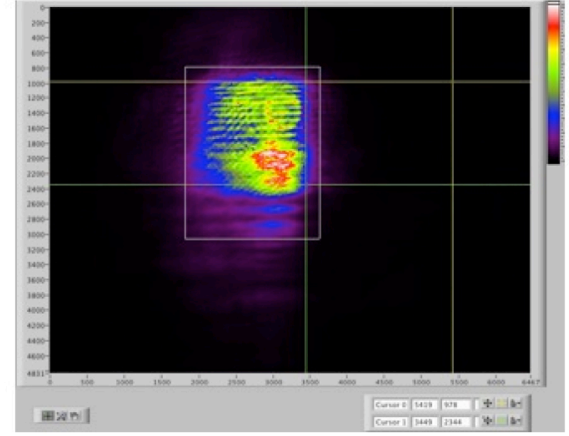

(b)

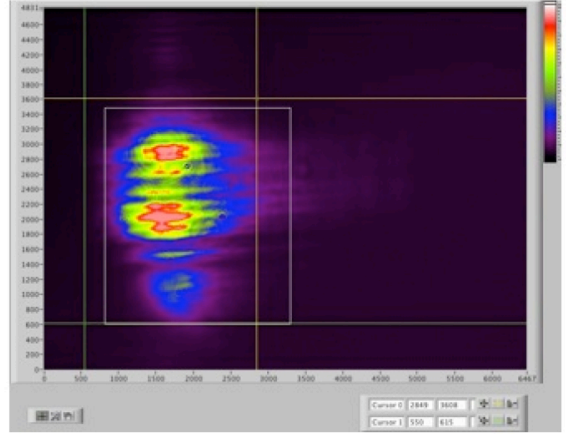

(c)

Figure 7 - HOMER optical performance: (a) single mode far field image, (b) intracavity HR image used for fluence monitoring, and (c) GRM output near field image. 
method caused any other system irregularities. No discernable effects were witnessed. Also seen in the data are slight dips and irregularities due to flip mirror misalignments after each MLA ranging test. For the bar drop event, the output energy dropped by $10 \%$ for a corresponding 3.6\% drop in pump energy. This matched our laser models very well and thus we were confident to proceed with the current increase. This was later verified under a post test inspection process. We finished the test with a diode current of $51.5 \mathrm{~A}$ and a pulse width of 88 us. The LDAs current started the experiment at a derating of $28.7 \%$ and completed the 2 billion shot run at $26.4 \%$. The pump pulsewidth was stepped up by $3.5 \%$ and produced another $10 \%$ in performance, very similar to the current step. Thus, an equivalent change of LDA drive current could be estimated confidently at $\sim 7 \%$, which includes the loss of a single bar. There is still $21.7 \%$ of headroom on the current for current corrections as the diodes decay.

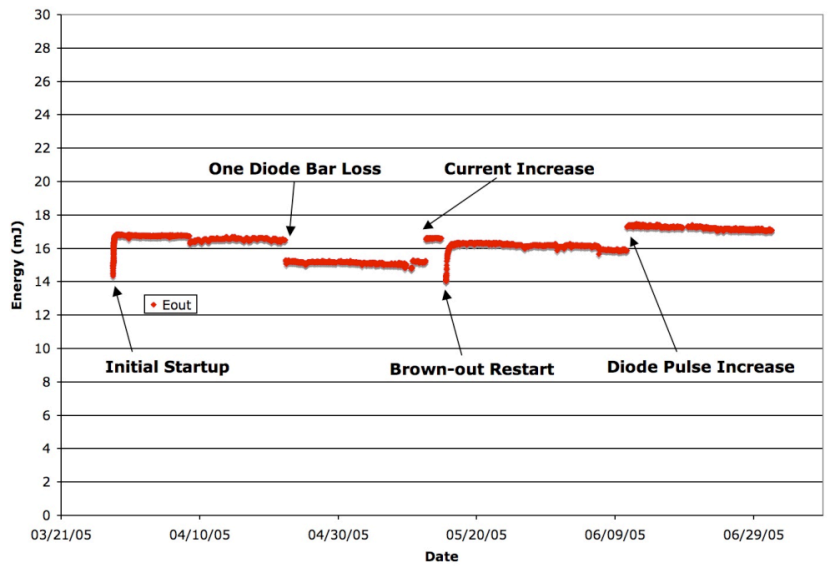

Figure 8 - Plot of output pulse energy of HOMER over 2 billion shots. Note the single bar drop, one electrical failure and 2 pump increases. Final pulse energy degradation is $0.94 \mathrm{~mJ} / 10^{9}$ shots.

\section{Conclusions}

The HOMER cavity is being studied further for any thermomechanical, opto-mechanical, and mass reduction improvements that can be made to produce a new version to be flight qualified. The $3^{\text {rd }}$ generation HOMER system described in this article was operated for an initial run $\sim 2$ billion shots with no damage and $\sim 7 \%$ degradation of diode laser performance including a single bar drop. The end of test performance still showed a $\sim 26.4 \%$ derated current setpoint on the LDAs with a degradation slope of $\sim 0.94$ $\mathrm{mJ} / 10^{9}$ shots. Making end-of-life estimates from a relatively short data run is not very reliable, but our confidence is high that this laser would safely go past 10 billion shots and still have significant headroom in LDA drive current. This estimate would more than fulfill the BioMM mission requirements of maintaining $17-15 \mathrm{~mJ}$ at $242 \mathrm{~Hz}$ for $\sim 5 \mathrm{x} 10^{9}$ laser pulses over 2 years. A $4^{\text {th }}$ generation HOMER laser is under development incorporating more lessons learned, from this and previous units, and will undergo full flight qualification tests. Plans and funding are being developed for eventual long-term, catastrophic life testing and power cycling in mid-late 2006 to determine its full potential for mission lifetime and suitability.

\section{BIOGRAPHY}

Barry Coyle is a laser physicist working at NASA's Goddard Space Flight Center. He received his PhD in 1992 from The American University through a grant with NASA

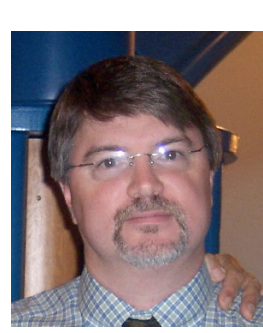
and has worked at GSFC ever since. He has developed and flown aircraft altimeter laser systems and designed and developed several candidate flight level systems for global vegetation measurement and planetary mapping. He also pursues research in alternative diode pumping designs and materials and is developing seeded fiber amplifiers for high-density planetary surface mapping and imaging.

Paul Stysley is a laser engineer working for American University on a grant at NASA-GSFC. He has developed several q-switched ND:YAG oscillators and amplifiers for

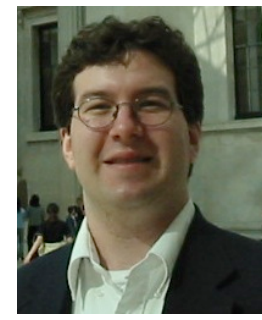
NASA's laser remote sensing efforts. $\mathrm{He}$ is also leading the design of an automated laser development data system for Apple Macintosh computers. He has a Masters degree in physics from the Catholic University of America. 


\section{REFERENCES}

[1] D. Barry Coyle, Richard B. Kay, Paul R. Stysley, Demetrios Poulios, "High Reliability, Long Life, Diode Pumped Nd:YAG Unstable Resonator for Space Based Vegetation Altimetry and Terrain Mapping," Appl. Optics. 43, 5236-5242, (2004).

[2] National Optics Institute, (NOI), 369 Franquet, Saint-Foy, Quebec, Canada G1p-4N8. M. Morin, technical consultant (morin@ino.qc.ca) See also "Graded Reflectivity mirror unstable laser resonators", Morin, M., Opt. And QE 29, 819-866, (1997).

[3] D. B. Coyle, R. B. Kay, P. R. Stysley, D. Poulios, "A Diode Pumped, Nd:YAG, Q-Switched Unstable Resonator Developed for Multi-Billion Shot, SpacedBased Remote Sensing Applications," CLEO/PHAST Conference Proceedings, (Baltimore, MD, May 2004)

[4] D. B. Coyle, "Design and Performance of the Vegetation Canopy Lidar (VCL) Laser Transmitter," IEEE International Aerospace Conference, Big Sky, MT. March. Proceedings, (2002).

[5] Peter Milonni and Joseph Eberly, Lasers, Wiley, 1988.

[6] E. Armandillo, C. Norrie, A. Cosentino, P. Laporta, P. Wazen, and P. Maine, "Diode-Pumped High Efficiency High-Brightness Q-switched Nd:YAG Slab Laser," Opt. Let. 22, 1168-1170, (1997).

[7] D. Krebs, A.M. Novo-Gradac, S. Li, S. Lindauer, R. Afzal, and A. Yu "Compact, Passively Q-switched Nd:YAG Laser for the Messenger Mission to Mercury" App. Opt. 44, 1715-1718, (2005).

[8] R. S. Afzal, A. W. Yu, J. J. Zayhowski, and T.Y. Fan, "Single-Mode High-Peak-Power Passively Q-switched Diode-Pumped Nd:YAG Laser," Opt. Let. 22, 1314-1316, (1997).

[9] T. J. Kane, R. C. Eckardt, and R.L. Byer, "Reduced thermal focusing and birefringence in zig-zag slab geometry crystalline lasers," IEEE JQE 19, 1351-1354, (1983).

[10] J. M. Eggleston, T. J. Kane, K. Kuhn, J. Unternahrer, and R. L. Byer, "The slab geometry laser-part I: theory," IEEE
JQE
20, 289-301,
(1984)

[11] X. Sun, G. A. Neumann, J. F. McGarry, T. W. Zagwodzki, J. F. Cavanaugh, J. J. Degnan, D. B. Coyle, D. R. Skillman, M. T. Zuber, and D. E. Smith, "Laser Ranging between the Mercury Laser Altimeter and an Earth-based Laser Satellite Tracking Station over a 24-million-km Distance", OSA Annual Meeting, Tuscon, AZ, Oct. Proceedings (2005) 